\title{
The role of postmastectomy radiotherapy in clinically node- positive, stage II-III breast cancer patients with pathological negative nodes after neoadjuvant chemotherapy: an analysis from the NCDB
}

\author{
Jieqiong Liu'1,2,*, Kai Mao ${ }^{3,4, *}$, Shuai Jiang ${ }^{5}$, Wen Jiang ${ }^{6}$, Kai Chen ${ }^{1,2}$, Betty Y.S. Kim7, \\ Qiang Liu ${ }^{1}$ and Lisa K. Jacobs ${ }^{2}$ \\ ${ }^{1}$ Guangdong Provincial Key Laboratory of Malignant Tumor Epigenetics and Gene Regulation, Breast Tumor Center, Sun Yat- \\ sen Memorial Hospital, Sun Yat-sen University, Guangzhou, China \\ ${ }^{2}$ Department of Surgery, Johns Hopkins University School of Medicine, Baltimore, MD, USA \\ ${ }^{3}$ Guangdong Provincial Key Laboratory of Malignant Tumor Epigenetics and Gene Regulation, Department of General Surgery, \\ Sun Yat-sen Memorial Hospital, Sun Yat-sen University, Guangzhou, China \\ ${ }^{4}$ Department of Medicine, Johns Hopkins University School of Medicine, Baltimore, MD, USA \\ ${ }^{5}$ Department of Epidemiology, Johns Hopkins Bloomberg School of Public Health, Baltimore, MD, USA \\ ${ }^{6}$ Department of Radiation Oncology, MD Anderson Cancer Center, Houston, TX, USA \\ 7 Department of Neurological Surgery, Mayo Clinic Florida, Jacksonville, FL, USA \\ * These authors have contributed equally to this work \\ Correspondence to: Lisa K. Jacobs, email: ljacob14@jhmi.edu
}

Jieqiong Liv, email: liujieqion01@hotmail.com

Keywords: breast cancer, postmastectomy radiotherapy, complete pathological nodal response, neoadjuvant chemotherapy, survival benefit

Received: September 10,2015 Accepted: November 26, $2015 \quad$ Published: December 18, 2015

\section{ABSTRACT}

Purpose: The role of postmastectomy radiotherapy (PMRT) in clinically nodepositive, stage II-III breast cancer patients with pathological negative nodes (ypNO) after neoadjuvant chemotherapy (NAC) remains controversial.

Methods: A total of 1560 clinically node-positive, stage II-III breast cancer patients treated with NAC and mastectomy who achieved ypNO between 1998 and 2009 in the National Cancer Database were analyzed. The effects of PMRT on overall survival (OS) for the entire cohort and multiple subgroups were evaluated. Imputation and propensity score matching were used as sensitivity analyses to minimize biases.

Results: Of the entire 1560 eligible patients, $903(57.9 \%)$ received PMRT and $657(42.1 \%)$ didn't. At a median follow-up of 56.0 months, no statistical difference was observed for OS between two groups by univariate and multivariate analyses ( $P$ $=0.120 ;$ HR 1.571, 95\% CI 0.839-2.943). On subgroup analyses, PMRT significantly improved OS in patients with clinical stage IIIB/IIIC disease, T3/T4 tumor, or residual invasive breast cancer after NAC $(P<0.05)$. This improvement in OS remained significant after sensitivity analyses for the propensity score-matched patients.

Conclusions: This study demonstrated that PMRT showed a heterogeneous effect in clinically node-positive, stage II-III breast cancer patients with ypNO following NAC. PMRT improved OS for patients with clinical stage IIIB/IIIC disease, T3/T4 tumor, or residual invasive breast tumor after NAC. In the absence of definitive conclusions from prospective studies, including the ongoing NSABP B-51 trial, our findings may help identify specific groups of women with clinically node-positive, stage II-III breast cancers who could benefit from PMRT after NAC. 


\section{INTRODUCTION}

The optimal patient selection criterion for postmastectomy radiotherapy (PMRT) in the management of breast cancer is a subject of ongoing debate. Previous randomized trials have established a clear guideline regarding the use PMRT in the setting of adjuvant chemotherapy [1-3]. However, whether PMRT can provide similar benefits in patients who had excellent pathological response after treatment with preoperative chemotherapy (NAC) is less clear. No results from prospective trials have been reported to evaluate PMRT's effect in the neoadjuvant setting. The available retrospective data suggest that the initial extent of disease clinically at presentation, the response of axillary lymph nodes to NAC, and the pathologic extent of residual disease are important factors to consider regarding the use of PMRT after NAC [4-6]. Thus, the National Cancer Institute (NCI) statement recommends that PMRT to the chest-wall and regional nodal basins should be considered for patients with clinical stage III disease or have histologically positive nodes after NAC [7]. Despite this, it remains unclear as to whether PMRT can provide improved patient outcomes for women with clinically node-positive, stage II to III breast cancer, but had a complete pathological nodal response (ypN0) after NAC. Previous studies aimed to address this question based on small retrospective cohorts have produced inconsistent results [4, 8-11]. A French group and another Korean study both showed that PMRT was not correlated with improved outcomes in clinical stage II-III patients with ypN0 after NAC $[8,10]$. In contrast, research conducted at MD Anderson Cancer Center found that PMRT significantly improved localregional recurrence (LRR) and cancer-specific survival (CSS) in clinical stage III breast cancer women even when they achieved a pathological complete response (pCR) following NAC $[4,11]$.

Therefore, there is a lack in consensus among practitioners regarding to the treatment recommendations of PMRT for clinically node-positive, stage II-III breast cancer patients with ypN0 after NAC. This was further demonstrated by a 2013 survey of 372 radiation oncologists which showed a split decision regarding treatment recommendations for clinical stage $\mathrm{T} 2 \mathrm{~N} 1$ patients who achieve ypN0 after NAC, with $49.9 \%$ of those surveyed recommending PMRT [12]. Given the conflicting results of small retrospective studies and lack of findings from randomized controlled trials, we analyzed a large national cohort of breast cancer patients, the National Cancer Database (NCDB), to identify the effectiveness of PMRT in terms of overall survival for clinically node-positive, stage II-III breast cancer patients with ypN0 after NAC.

\section{MATERIALS AND METHODS}

\section{Patient population}

We used data from the NCDB, which is a national hospital-based cancer registry jointly sponsored by the American College of Surgeons and the American Cancer Society, and collects data on about $70 \%$ of newly diagnosed breast cancer cases in the United States. Data are coded and reported according to nationally established protocols coordinated under the auspices of the North American Association of Central Cancer Registries.

Data within the NCDB were rendered anonymous, so the study was exempt from review by the Johns Hopkins Medicine Institutional Review Board, and no consent was needed in this study.

A total of 2,807,541 breast cancer cases (International Classification of Diseases for Oncology, 3rd edition [ICD-O-3] histology codes 8000-8576, 89808981, and 9020/3 [13]), diagnosed between 1998 and 2012 , were identified. The inclusion criteria were women 18 years or older, clinically node-positive and stage II-III (AJCC) breast cancer, treated with NAC and mastectomy with pathologically confirmed complete nodal response (ypN0). To ensure adequate follow-up time, we included cases diagnosed from 1998 through 2009. The timing of chemotherapy is coded in the NCDB as a temporal sequence with relation to definitive surgical therapy, allowing the accurate identification of NAC. Patients with positive or unknown surgical margin, pathological tumor size $>5 \mathrm{~cm}$ after NAC, distant metastatic disease, or prior malignancy were excluded. Additional exclusion criteria included unknown clinical or pathological tumor/ node stage, preoperative or intraoperative radiotherapy, or radiotherapy not for chest wall and draining lymphatics. This resulted in a cohort of 1580 patients of which 907 received PMRT and 673 patients did not. The primary endpoint for this study was overall survival (OS), which is defined as the time from diagnosis to the date of death from any cause. Some patients did not receive radiotherapy after surgery because of rapid death (due to disease progression or post-operative complications) or loss of follow-up. To minimize this potential bias between groups, we excluded patients who died or lost to followup within 3 months after mastectomy: $4(0.4 \%)$ in the irradiated group and $16(2.4 \%)$ in the non-irradiated group. This left 1560 patients for final analysis.

\section{Statistical analysis}

\section{Primary analysis}

Characteristics of the entire study population were presented according to PMRT treatment. The demographic 
and clinicopathological characteristics were compared between the two groups using the $\chi^{2}$ test. OS curves were constructed using the Kaplan-Meier method and compared between the two groups using the log-rank test. Multivariate Cox proportional hazard model was applied to assess the independent prognostic effect of PMRT or other factors. Likelihood ratio test was used to select the best multivariate Cox model. We also performed subgroup analyses to identify the role of PMRT on OS in various subgroups of patients.

\section{Sensitivity analysis using imputation and propensity score}

Propensity score-based sensitivity analysis was done to minimize selection bias or a lack of covariate balance. In the NCDB database, some key variables (eg. histologic grade) contain missing data, which may result in biases. To compensate for this, multiple imputation methods by chained equations [14-16] to account for the missing values of several variables was performed before the propensity score matching. NCDB has neither ER/PR records before 2004, nor Chalson/Deyo score before 2003, we can not assume unknown ER/PR status and Chalson/ Deyo score are missing at random. Thus, we conducted the imputation to accommodate missing data for insurance status, histologic grade, number of examined lymph nodes, chemotherapy type, and use of hormone therapy, but not for ER/PR status or Chalson/Deyo score. A probabilistic rule based on regression models for each covariable with the other covariables serving as predictors was used to impute possible values for individual missing values. A full dataset was created after imputing for 10 times using "complete" function in MICE package $[16,17]$.

For the entire study cohort and individual subgroups, we performed logistic regression to select demographic and clinicopathological variables associated with the implementation of PMRT. All variables with a univariate $P$ value $\leq 0.20$ were eligible for inclusion in the logistic regression model. The final multivariate logistic model was used to calculate the propensity score for each individual, which is the probability of the patient being treated with PMRT. Patients who received PMRT were matched to patients who did not receive PMRT by propensity score \pm 0.1 in a $1: 1$ ratio. The quality of the matching was checked by calculating the standardized difference for each covariate, assuming that the balance was achieved if the standardized difference was less than 0.1 [18]. Univariate and/or multivariate survival analyses were performed in the propensity score-matched populations using the same methods as those in the primary analysis.

Statistical analyses were conducted using STATA 12.0 software (StataCrop, College Station, TX) or R software (R Core Team 2014 [19]). All statistical tests were two-sided, and statistical significance was defined as $P<0.05$.

\section{RESULTS}

\section{Patient and treatment characteristics}

Of the 1560 clinically node-positive, stage II-III breast cancer patients who had complete pathological nodal response after NAC and mastectomy, 903 (57.9\%) received PMRT and 657 (42.1\%) did not. All the patients had negative surgical margins. Table 1 presents the

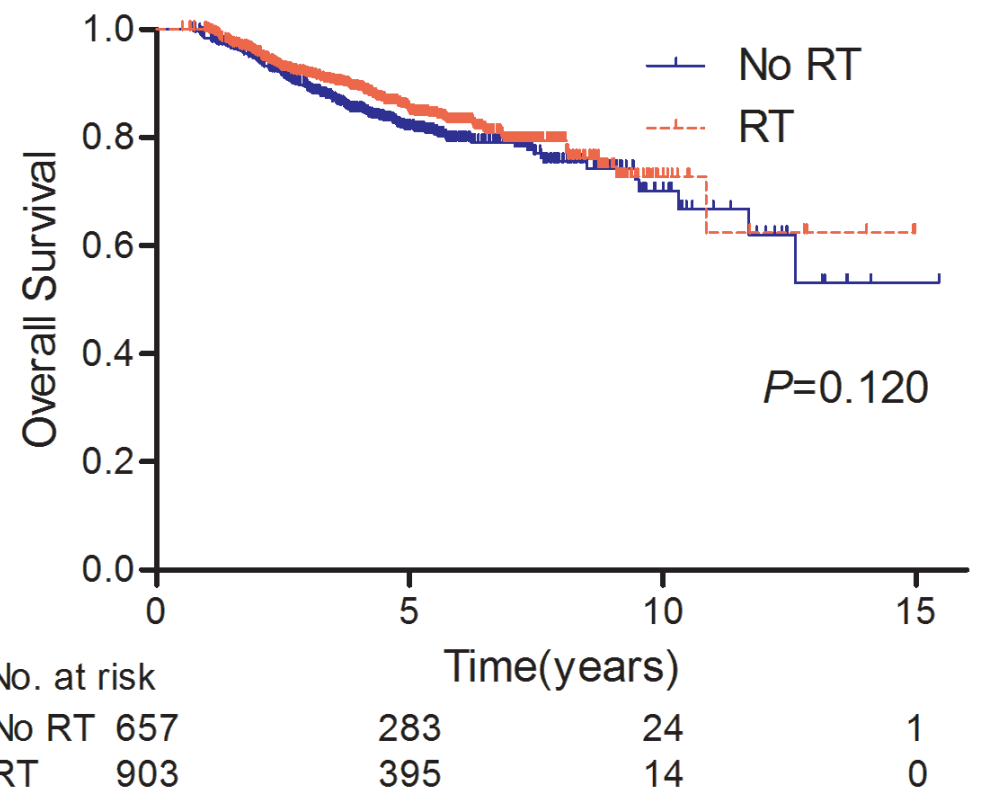

Figure 1: Rate of overall survival for the entire cohort of patients treated with PMRT $(n=903)$ and without PMRT $(n=657)$. 
Table 1: Characteristics of the whole study population $(n=1560)$

\begin{tabular}{|c|c|c|c|c|c|}
\hline \multirow[t]{2}{*}{ Characteristics } & \multicolumn{2}{|c|}{$\begin{array}{l}\text { No PMRT } \\
(n=657)\end{array}$} & \multicolumn{2}{|c|}{$\begin{array}{l}\text { PMRT } \\
(n=903)\end{array}$} & \multirow[t]{2}{*}{$P$} \\
\hline & No. & $\%$ & No. & $\%$ & \\
\hline Age, years & & & & & $\mathrm{NS}$ \\
\hline Median (range) & \multicolumn{2}{|c|}{$50(20-86)$} & \multicolumn{2}{|c|}{$50(22-88)$} & \\
\hline$\leq 40$ & 143 & 21.8 & 203 & 22.5 & \\
\hline $41-60$ & 386 & 58.7 & 545 & 60.3 & \\
\hline$>60$ & 128 & 19.5 & 155 & 17.2 & \\
\hline Race & & & & & $\mathrm{NS}$ \\
\hline White & 494 & 75.2 & 693 & 76.7 & \\
\hline Black & 121 & 18.4 & 162 & 17.9 & \\
\hline Asian or other & 42 & 6.4 & 48 & 5.3 & \\
\hline Insurance status & & & & & NS \\
\hline Not insured & 31 & 4.7 & 49 & 5.4 & \\
\hline Private insurance & 426 & 64.8 & 620 & 68.7 & \\
\hline Public insurance & 186 & 28.3 & 227 & 25.1 & \\
\hline Unknown & 14 & 2.1 & 7 & 0.8 & \\
\hline Chalson/Deyo score & & & & & $<0.001$ \\
\hline 0 & 552 & 84.0 & 797 & 88.3 & \\
\hline 1 & 44 & 6.7 & 83 & 9.2 & \\
\hline 2 & 10 & 1.5 & 7 & 0.8 & \\
\hline Unknown & 51 & 7.8 & 16 & 1.8 & \\
\hline Year of diagnosis & & & & & $<0.001$ \\
\hline $1998-2003$ & 90 & 13.7 & 56 & 6.2 & \\
\hline $2004-2009$ & 567 & 86.3 & 847 & 93.8 & \\
\hline \multicolumn{6}{|l|}{ Histological type } \\
\hline Ductal & 540 & 82.2 & 718 & 79.6 & $\mathrm{NS}$ \\
\hline Lobular & 50 & 7.6 & 61 & 6.7 & \\
\hline Other & 67 & 10.2 & 124 & 13.7 & \\
\hline No. of nodes examined & & & & & 0.009 \\
\hline Median (range) & \multicolumn{2}{|c|}{$11(1-46)$} & \multicolumn{2}{|c|}{$12(1-46)$} & \\
\hline $1-10$ & 317 & 48.2 & 379 & 42.0 & \\
\hline$>10$ & 320 & 48.7 & 507 & 56.1 & \\
\hline Unknown & 20 & 3.0 & 17 & 1.9 & \\
\hline Clinical T-stage & & & & & $<0.001$ \\
\hline $\mathrm{T} 1$ & 79 & 12.0 & 55 & 6.1 & \\
\hline $\mathrm{T} 2$ & 276 & 42.0 & 254 & 28.1 & \\
\hline T3 & 170 & 25.9 & 279 & 30.9 & \\
\hline T4 & 132 & 20.1 & 315 & 34.9 & \\
\hline Clinical N-stage & & & & & $<0.001$ \\
\hline N1 & 530 & 80.7 & 651 & 72.1 & \\
\hline N2 & 90 & 13.7 & 161 & 17.8 & \\
\hline N3 & 37 & 5.6 & 91 & 10.1 & \\
\hline Clinical AJCC stage & & & & & $<0.001$ \\
\hline II & 325 & 49.5 & 231 & 25.6 & \\
\hline III & 332 & 50.5 & 672 & 74.4 & \\
\hline Pathologic T-stage (after NAC) & & & & & NS \\
\hline T0/Tis & 277 & 42.2 & 399 & 44.2 & \\
\hline $\mathrm{T} 1$ & 221 & 33.6 & 315 & 34.9 & \\
\hline $\mathrm{T} 2$ & 159 & 24.2 & 189 & 20.9 & \\
\hline Histologic grade & & & & & NS \\
\hline
\end{tabular}




\begin{tabular}{|l|l|l|l|l|l|l|}
\hline Well or moderately & 161 & 24.5 & & 199 & 22.0 & \\
\hline Poorly or undifferentiated & 413 & 62.9 & & 613 & 67.9 & \\
\hline Unknown & 83 & 12.6 & & 91 & 10.1 & \\
\hline ER* & & & & & & $<0.001$ \\
\hline Negative & 330 & 50.2 & & 503 & 55.7 & \\
\hline Positive & 208 & 31.7 & & 331 & 36.7 & \\
\hline Unknown & 119 & 18.1 & & 69 & 7.6 & \\
\hline PR* & & & & & & $<0.001$ \\
\hline Negative & 379 & 57.7 & & 563 & 62.3 & \\
\hline Positive & 159 & 24.2 & & 270 & 29.9 & \\
\hline Unknown & 119 & 18.1 & & 70 & 7.8 & \\
\hline Chemotherapy type & & & & & & $<0.001$ \\
\hline Single-agent & 13 & 2.0 & & 4 & 0.4 & \\
\hline Multi-agent & 588 & 89.5 & & 881 & 97.6 & \\
\hline Unknown if single or multi- & 56 & 8.5 & & 18 & 2.0 & \\
\hline Hormone therapy & & & & & & $<0.001$ \\
\hline No & 449 & 68.3 & & 539 & 59.7 & \\
\hline Yes & 181 & 27.5 & & 335 & 37.1 & \\
\hline Unknown & 27 & 4.1 & & 29 & 3.2 & \\
\hline
\end{tabular}

Abbreviations: PMRT, postmastectomy radiotherapy; NS, not significant $(P>0.05)$;

AJCC, American Joint Committee on Cancer (fifth or sixth edition); NAC, neoadjuvant chemotherapy; ER, estrogen receptor; PR, progesterone receptor

*ER or PR groups include those with borderline results.

comparisons of demographic, clinicopathological, and treatment characteristics between these two cohorts of patients. When compared with patients who did not receive PMRT, irradiated patients had less comorbidities, more advanced clinical tumor stage, nodal stage, or AJCC stage, more regional lymph nodes examined, and less unknown ER/PR status, and received more multiagent chemotherapy or hormone therapy $(P<0.01$ for all comparisons). No difference was found between the two groups with respect to age, race, insurance status, pathological tumor stage (after NAC), or histologic grade. For the patients treated with PMRT, radiation targets included chest wall and draining lymphatics, with or without a chest wall boost. The median dose of radiation was $50.4 \mathrm{~Gy}$.

\section{Survival analyses for the whole population}

Overall, the median follow-up was 56.0 months (range, 6.14-185.4 months). At the cutoff date for the survival analysis (December 2013), a total of 139 (15.4\%) and $124(18.9 \%)$ patients died in the PMRT and no PMRT group, respectively. The 5-year OS rates in the two groups were not significantly different $(84.6 \%$ for PMRT vs $81.7 \%$ for no PMRT, $P=0.120$, Figure 1). PMRT also showed no association with a difference in OS by multivariate analysis (PMRT vs no PMRT: HR 0.820, 95\% CI 0.630-1.068, Table 2). Factors found to be significant
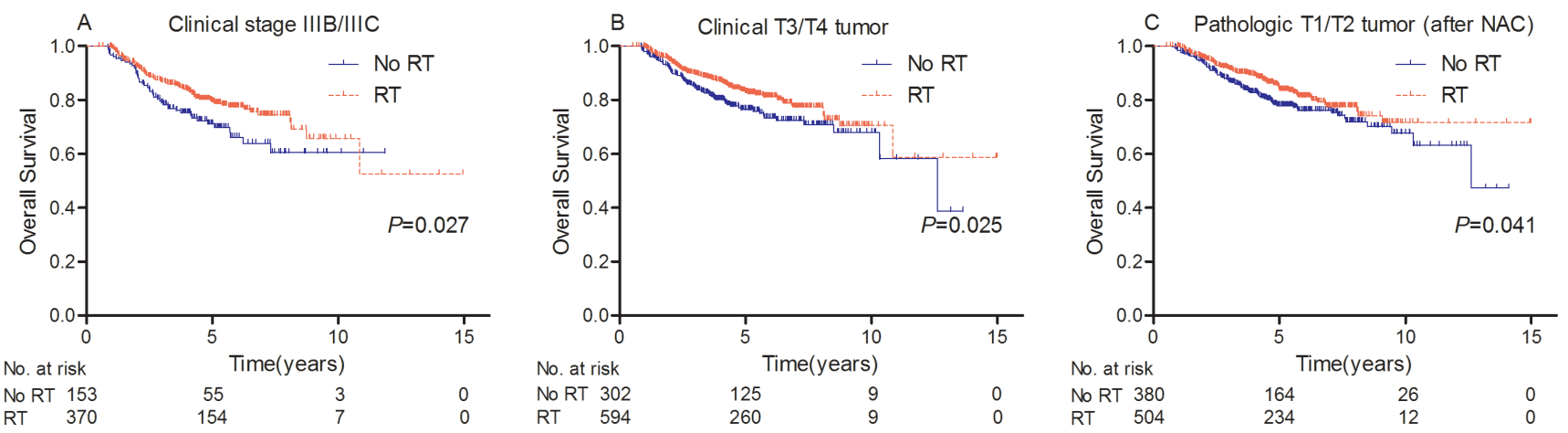

Figure 2: Rate of overall survival for patients with A. clinical IIIB/IIIC disease, B. clinical T3/T4 tumor, or C. pathologic T1/T2 tumor after NAC who were treated with PMRT and without PMRT. 
Table 2: Multivariate analysis of OS for the whole study population $(n=1560)$

\begin{tabular}{|c|c|c|c|}
\hline Factors & HR & $95 \% \mathrm{CI}$ & $P$ \\
\hline \multicolumn{4}{|l|}{ Age, years } \\
\hline$\leq 40$ & Reference & & \\
\hline $41-60$ & 1.209 & $0.857-1.706$ & 0.281 \\
\hline$>60$ & 1.692 & $1.122-2.553$ & 0.012 \\
\hline \multicolumn{4}{|l|}{ Race } \\
\hline White & Reference & & \\
\hline Black & 0.965 & $0.701-1.329$ & 0.829 \\
\hline Asian or other & 0.394 & $0.174-0.894$ & 0.026 \\
\hline \multicolumn{4}{|l|}{ Insurance status } \\
\hline Private insurance & Reference & & \\
\hline Public insurance & 1.468 & $1.093-1.971$ & 0.011 \\
\hline Not insured & 1.155 & $0.645-2.068$ & 0.627 \\
\hline Unknown & 1.176 & $0.423-3.270$ & 0.756 \\
\hline \multicolumn{4}{|l|}{ Histologic grade } \\
\hline Well differentiated & Reference & & \\
\hline Moderately differentiated & 9.749 & $1.331-71.425$ & 0.025 \\
\hline Poorly or undifferentiated & 7.760 & $1.066-56.489$ & 0.043 \\
\hline Unknown & 9.221 & $1.239-68.657$ & $\mathbf{0 . 0 3 0}$ \\
\hline \multicolumn{4}{|c|}{ Examined regional nodes number } \\
\hline $0-10$ & Reference & & \\
\hline$>10$ & 0.770 & 0.598-0.991 & 0.043 \\
\hline Unkown & 1.196 & $0.576-2.482$ & 0.631 \\
\hline \multicolumn{4}{|l|}{ Clinical T-stage } \\
\hline $\mathrm{T} 1$ & Reference & & \\
\hline $\mathrm{T} 2$ & 0.692 & 0.419-1.141 & 0.149 \\
\hline T3 & 1.575 & $0.784-3.167$ & 0.202 \\
\hline $\mathrm{T} 4$ & 2.808 & $1.395-5.649$ & 0.004 \\
\hline \multicolumn{4}{|l|}{ Clinical AJCC stage } \\
\hline II & Reference & & \\
\hline III & 2.193 & $1.197-4.017$ & 0.011 \\
\hline \multicolumn{4}{|c|}{ Pathologic T-stage (after NAC) } \\
\hline T0/Tis & Reference & & \\
\hline $\mathrm{T} 1$ & 1.275 & $0.943-1.724$ & 0.115 \\
\hline $\mathrm{T} 2$ & 1.599 & $1.160-2.205$ & 0.004 \\
\hline \multicolumn{4}{|l|}{ Hormone therapy } \\
\hline No & Reference & & \\
\hline Yes & 0.647 & $0.441-0.951$ & $\mathbf{0 . 0 2 7}$ \\
\hline Unknown & 0.618 & $0.300-1.273$ & 0.192 \\
\hline \multicolumn{4}{|l|}{ PMRT } \\
\hline No & Reference & & \\
\hline Yes & 0.820 & $0.630-1.068$ & 0.141 \\
\hline
\end{tabular}

Abbreviations: OS, overall survival; HR, hazard ratio; CI, confidence interval; AJCC, American Joint Committee on Cancer (fifth or sixth edition); NAC, neoadjuvant chemotherapy; PMRT, postmastectomy radiotherapy

Table 3: Subgroup analyses for the effect of PMRT on the 5-year OS rate in the primary analysis

\begin{tabular}{|l|l|l|l|}
\hline \multirow{2}{*}{ Factors } & 5-year OS Rate (\%) & \multirow{2}{*}{$\boldsymbol{P}$} \\
\cline { 2 - 4 } & No PMRT & PMRT & \\
\hline Age & & & \\
\hline$\leq 40$ & 90.2 & 86.7 & 0.136 \\
\hline $41-60$ & 82.8 & 85.4 & 0.151 \\
\hline$>60$ & 67.8 & 78.9 & 0.053 \\
\hline
\end{tabular}




\begin{tabular}{|l|l|l|l|}
\hline Clinical AJCC stage & & & \\
\hline II & 83.9 & 86.5 & 0.424 \\
\hline IIIA & 86.8 & 89.5 & 0.247 \\
\hline IIIB/IIIC & 71.2 & 79.3 & $\mathbf{0 . 0 2 7}$ \\
\hline Clinical T-stage & & & \\
\hline T1/T2 & 86.0 & 87.8 & 0.329 \\
\hline T3/T4 & 76.6 & 82.8 & $\mathbf{0 . 0 2 5}$ \\
\hline Clinical N-stage & & & \\
\hline N1 & 81.7 & 84.8 & 0.191 \\
\hline N2/N3 & 81.5 & 84.0 & 0.359 \\
\hline Pathologic T-stage (after NAC) & & & \\
\hline T0/Tis & 86.9 & 86.0 & 0.891 \\
\hline T1/T2 & 78.0 & 83.6 & $\mathbf{0 . 0 4 1}$ \\
\hline Hormone receptor* & & & \\
\hline Negative & 82.6 & 83.4 & 0.587 \\
\hline Positive & 82.9 & 87.6 & 0.178 \\
\hline
\end{tabular}

Abbreviations: PMRT, postmastectomy radiotherapy; OS, overall survival; AJCC, American Joint Committee on Cancer (fifth or sixth edition); NAC, neoadjuvant chemotherapy

*We created a joint hormone receptor status using estrogen receptor (ER) and progesterone receptor (PR) status. Those with either ER or PR positive status (ER or PR positive groups included those with borderline results) were grouped as hormone receptor positive, and those with ER and PR negative status were grouped as hormone receptor negative.

Table 4: Characteristics and standardised mean differences of covariates among patients with or without PMRT before and after propensity-score matching

\begin{tabular}{|c|c|c|c|c|c|c|}
\hline \multirow[b]{2}{*}{ Characteristics } & \multicolumn{3}{|c|}{ Before matching $(n=1560)$} & \multicolumn{3}{|c|}{ After mathching $(n=1046)$} \\
\hline & $\begin{array}{l}\text { No PMRT } \\
(\%)\end{array}$ & $\begin{array}{l}\text { PMRT } \\
(\%)\end{array}$ & $\begin{array}{l}\text { Std. mean } \\
\text { difference }\end{array}$ & $\begin{array}{l}\text { No PMRT } \\
(\%)\end{array}$ & $\begin{array}{l}\text { PMRT } \\
(\%)\end{array}$ & $\begin{array}{l}\text { Std. mean } \\
\text { difference }\end{array}$ \\
\hline \multicolumn{7}{|l|}{ Age, years } \\
\hline$\leq 40$ & 21.8 & 22.5 & 0.017 & 22.0 & 22.7 & 0.018 \\
\hline $41-60$ & 58.7 & 60.3 & 0.032 & 58.5 & 60.4 & 0.039 \\
\hline$>60$ & 19.5 & 17.2 & -0.060 & 19.5 & 16.9 & -0.069 \\
\hline \multicolumn{7}{|l|}{ Race } \\
\hline White & 75.2 & 76.7 & 0.036 & 75.7 & 74.0 & -0.041 \\
\hline Black & 18.4 & 17.9 & -0.012 & 18.4 & 20.5 & 0.055 \\
\hline Asian or other & 6.4 & 5.3 & -0.047 & 5.9 & 5.5 & -0.017 \\
\hline \multicolumn{7}{|l|}{ Insurance status } \\
\hline Not insured & 5.1 & 5.6 & 0.054 & 5.7 & 5.3 & 0.017 \\
\hline Private insurance & 66.4 & 68.9 & 0.020 & 66.9 & 67.7 & -0.017 \\
\hline Public insurance & 28.5 & 25.5 & -0.069 & 27.4 & 27.0 & -0.009 \\
\hline \multicolumn{7}{|l|}{ Chalson/Deyo score } \\
\hline 0 & 84.0 & 88.3 & 0.132 & 87.6 & 87.2 & -0.012 \\
\hline 1 & 6.7 & 9.2 & 0.086 & 7.5 & 8.6 & 0.040 \\
\hline 2 & 1.5 & 0.8 & -0.085 & 1.1 & 1.1 & 0.000 \\
\hline Unknown & 7.8 & 1.8 & -0.454 & 3.8 & 3.1 & -0.058 \\
\hline \multicolumn{7}{|l|}{ Year of diagnosis } \\
\hline $1998-2003$ & 13.7 & 6.2 & -0.311 & 9.2 & 8.6 & -0.024 \\
\hline $2004-2009$ & 86.3 & 93.8 & 0.311 & 90.8 & 91.4 & 0.024 \\
\hline \multicolumn{7}{|l|}{ Histological type } \\
\hline Ductal & 82.2 & 79.6 & -0.064 & 81.2 & 81.4 & 0.005 \\
\hline Lobular & 7.6 & 6.7 & -0.039 & 7.3 & 7.3 & 0.000 \\
\hline Other & 10.2 & 13.7 & 0.103 & 11.5 & 11.3 & -0.006 \\
\hline \multicolumn{7}{|c|}{ No. of nodes examined } \\
\hline $1-10$ & 49.8 & 42.9 & -0.140 & 49.0 & 45.1 & -0.077 \\
\hline$>10$ & 50.2 & 57.1 & 0.140 & 51.0 & 54.9 & 0.077 \\
\hline
\end{tabular}




\begin{tabular}{|c|c|c|c|c|c|c|}
\hline \multicolumn{7}{|l|}{ Clinical T-stage } \\
\hline T1 & 12.0 & 6.1 & -0.248 & 8.8 & 8.6 & -0.008 \\
\hline $\mathrm{T} 2$ & 42.0 & 28.1 & -0.309 & 39.0 & 39.2 & 0.004 \\
\hline T3 & 25.9 & 30.9 & 0.109 & 28.5 & 27.9 & -0.012 \\
\hline $\mathrm{T} 4$ & 20.1 & 34.9 & 0.310 & 23.7 & 24.3 & 0.012 \\
\hline \multicolumn{7}{|l|}{ Clinical N-stage } \\
\hline N1 & 80.7 & 72.1 & -0.191 & 76.9 & 76.9 & 0.000 \\
\hline $\mathrm{N} 2$ & 13.7 & 17.8 & 0.108 & 16.0 & 14.5 & -0.040 \\
\hline N3 & 5.6 & 10.1 & 0.148 & 7.1 & 8.6 & 0.051 \\
\hline \multicolumn{7}{|l|}{ Clinical AJCC stage } \\
\hline II & 49.5 & 25.6 & -0.547 & 41.9 & 40.3 & -0.035 \\
\hline III & 50.5 & 74.4 & 0.547 & 58.1 & 59.7 & 0.035 \\
\hline \multicolumn{7}{|l|}{$\begin{array}{l}\text { Pathologic T-stage (after } \\
\text { NAC) }\end{array}$} \\
\hline T0/Tis & 42.2 & 44.2 & 0.041 & 43.2 & 40.9 & -0.046 \\
\hline $\mathrm{T} 1$ & 33.6 & 34.9 & 0.026 & 33.7 & 34.0 & 0.008 \\
\hline $\mathrm{T} 2$ & 24.2 & 20.9 & -0.080 & 23.1 & 25.1 & 0.047 \\
\hline \multicolumn{7}{|l|}{ Histologic grade } \\
\hline Well & 2.7 & 2.7 & -0.005 & 2.5 & 2.9 & 0.024 \\
\hline Moderatedly & 25.3 & 22.1 & -0.075 & 26.0 & 25.6 & -0.009 \\
\hline Poorly or undifferentiated & 72.0 & 75.2 & 0.074 & 71.5 & 71.5 & 0.000 \\
\hline \multicolumn{7}{|c|}{\begin{tabular}{|l|l} 
ER* $^{*}$ & \\
\end{tabular}} \\
\hline Negative & 50.2 & 55.7 & 0.110 & 53.7 & 51.8 & -0.038 \\
\hline Positive & 31.7 & 36.7 & 0.104 & 34.6 & 37.1 & 0.052 \\
\hline Unknown & 18.1 & 7.6 & -0.394 & 11.7 & 11.1 & -0.022 \\
\hline \multicolumn{7}{|l|}{$\mathrm{PR}^{*}$} \\
\hline Negative & 57.7 & 62.3 & 0.096 & 61.2 & 59.5 & -0.035 \\
\hline Positive & 24.2 & 29.9 & 0.124 & 27.2 & 29.2 & 0.046 \\
\hline Unknown & 18.1 & 7.8 & -0.387 & 11.6 & 11.3 & -0.014 \\
\hline \multicolumn{7}{|l|}{ Chemotherapy type } \\
\hline \begin{tabular}{|l} 
Single-agent \\
\end{tabular} & 3.0 & 0.5 & -0.391 & 0.6 & 0.8 & 0.029 \\
\hline Multi-agent & 97.0 & 99.5 & 0.391 & 99.4 & 99.2 & -0.029 \\
\hline
\end{tabular}

Abbreviations: PMRT, postmastectomy radiotherapy; NS, not significant $(P>0.05)$;

AJCC, American Joint Committee on Cancer (fifth or sixth edition); NAC, neoadjuvant chemotherapy; ER, estrogen receptor; $\mathrm{PR}$, progesterone receptor

*ER or PR groups include those with borderline results.

for worse OS by multivariate analysis included: age older than 60 years, white or black race, public insurance (compared with private insurance), higher histologic grade, fewer than 10 axillary nodes examined, clinical T4 tumor, clinical stage III disease, residual pathologic T2 tumor, and lack of hormone therapy $(P<0.05$ for all comparisons, Table 2).

However, subgroup analyses demonstrated PMRT significantly improved OS in patients with clinical stage IIIB/IIIC disease or T3/T4 tumor, or residual invasive breast tumor after NAC $(P<0.05$ for all comparisons; Table 3, Figure 2A to $2 \mathrm{C}$ ).

\section{Survival analyses for propensity score-matched populations}

Since patients who received PMRT differed from patients who did not receive PMRT, we performed propensity score analysis as a sensitivity analysis to ensure that previous results were not due to lack of baseline covariate balance. Before the propensity score analysis, we used multiple imputation methods to account for the missing values of several variables. Sensitivity analysis showed that the effects of PMRT on OS were quite similar before and after imputation (data not shown).

Of the entire study population, we matched 523 pairs of patients by propensity scores. Table 4 shows the characteristics and standardized mean differences of all covariates between the two groups before and 
Table 5: Subgroup analyses for the effect of PMRT on the 5-year OS rate in the sensitivity analysis (for the propensity score-matched patients)

\begin{tabular}{|l|l|l|l|}
\hline \multirow{2}{*}{ Factors } & \multicolumn{2}{|l|}{ 5-year OS Rate (\%) } & \multirow{2}{*}{$\boldsymbol{P}$} \\
\cline { 2 - 4 } & No PMRT & PMRT & \\
\hline Age & & & \\
\hline$\leq 40$ & 92.3 & 87.4 & 0.296 \\
\hline $41-60$ & 82.8 & 86.8 & 0.076 \\
\hline$>60$ & 67.8 & 77.8 & 0.138 \\
\hline Clinical AJCC stage & & & \\
\hline II & 80.7 & 86.0 & 0.236 \\
\hline IIIA & 53.1 & 51.9 & 0.687 \\
\hline IIIB/IIIC & 71.6 & 80.4 & $\mathbf{0 . 0 4 6}$ \\
\hline Clinical T-stage & & & \\
\hline T1/T2 & 85.1 & 85.5 & 0.420 \\
\hline T3/T4 & 76.9 & 81.7 & $\mathbf{0 . 0 4 9}$ \\
\hline Clinical N-stage & & & \\
\hline N1 & 83.4 & 84.9 & 0.247 \\
\hline N2/N3 & 81.2 & 80.3 & 0.946 \\
\hline Pathologic T-stage (after NAC) & & & \\
\hline T0/Tis & 91.2 & 86.0 & 0.645 \\
\hline T1/T2 & 78.0 & 84.6 & $\mathbf{0 . 0 3 2}$ \\
\hline Hormone receptor* & & & \\
\hline Negative & 83.7 & 80.4 & 0.580 \\
\hline Positive & 81.5 & 85.6 & 0.352 \\
\hline
\end{tabular}

Abbreviations: PMRT, postmastectomy radiotherapy; OS, overall survival; AJCC, American Joint Committee on Cancer (fifth or sixth edition); NAC, neoadjuvant chemotherapy

*We created a joint hormone receptor status using estrogen receptor (ER) and progesterone receptor (PR) status. Those with either ER or PR positive status (ER or PR positive groups included those with borderline results) were grouped as hormone receptor positive, and those with ER and PR negative status were grouped as hormone receptor negative.

after matching. Univariate and multivariate analyses consistently showed no statistical effect of PMRT on OS $(P=0.167$; PMRT vs no PMRT: HR $0.847,95 \%$ CI $0.632-1.136$, Supplementary Figure 1, and Supplementary Table 1). We also matched the patients who received PMRT to those who did not, by propensity scores among distinct subgroups of patients based on the above primary subgroup survival analyses. Subgroup analyses for propensity score-matched populations identified PMRT improved OS in patients with clinical stage IIIB/IIIC disease, T3/T4 tumor, or residual invasive breast cancer after NAC, which is the same subset of patients as those defined by the primary subgroup analyses $(P<0.05$ for all comparisons; Table 5, Supplementary Figure $2 \mathrm{~A}$ to $2 \mathrm{C}$ ).

\section{DISCUSSION}

NAC is being used more frequently for clinical stage II or III breast cancer patients, raising issues regarding to the subsequent locoregional treatment, such as radiotherapy and sentinel node biopsy. However, the role of PMRT in clinically node-positive, stage II-III patients with negative pathological nodes (ypN0) after NAC, remains unclear. The current study included a large, registry-based, national patient cohort, with the aim to address this question. Our findings suggest that PMRT improves patient OS, but its benefit appears to be limited to selected patients with clinical stage IIIB/IIIC disease, clinical T3/T4 tumor, or residual invasive breast tumor after NAC.

One of the main effects of NAC is its potential to downstage the pathological extent of disease. Previous studies showed $20 \%$ to $40 \%$ of breast cancer patients with clinically positive nodes at diagnosis can achieve a complete pathological nodal response after NAC $[20,21]$. This pathological downstaging presents a unique challenge to treatment decision-making. Further complicating the issue is the lack of definitive data in the literature. Studies regarding the effectiveness of PMRT among women with clinically node-positive, stage II-III disease who downstaged to ypN0 following NAC were all based on retrospective analysis of small patient cohorts, and had conflicting results. A study from MD Anderson Cancer Center found that PMRT reduced LRR for patients with clinical stage III or IV disease and subsequently achieved a pCR to NAC, but no difference in LRR rates was observed in patients with clinical stage I-II disease with a pCR [4]. Their updated single-institutional experience, 
including 106 patients with a pCR in the breast and regional lymph nodes to NAC, indicated similar results (selected patients with clinical stage III disease with a pCR after NAC can benefit from PMRT) [11]. However, a French study showed that PMRT was not associated with improved local recurrence-free survival, diseasefree survival or OS in women with ypN0 after NAC [9]. Similarly, a multicenter retrospective study $(n=151)$ also reported that PMRT had no effectiveness in clinical stage II-III Korean breast cancer patients with ypN0 following NAC[10]. Since radiotherapy for breast cancer has toxicities including cardiac complications, pneumonitis, and lymphedema [22], and PMRT may lead to additional plastic surgeries for completion of breast reconstruction, the authors recommended the omission of PMRT in clinical stage II-III patients with ypN0 after NAC. Our study, based on a large patient cohort, on the other hand, is in support with the observations made by the MD Anderson studies. Unlike the French and Korean study, which had inherent shortcomings such as limited sample size, and multiple unbalanced baseline characteristics between groups, the current study represent the largest and utilized the most contemporary analysis to address this issue. The large sample size and more robust sensitivity analyses using imputation and propensity score matching enabled us to better quantify the survival benefit of PMRT for multiple subgroups of patients with minimal potential biases.

Clinically node-positive, stage II-III breast cancer patients who had complete nodal response to NAC after mastectomy represent a heterogeneous collection of patients with a wide range of demographic, clinicopathologic and treatment response related characteristics. Therefore, an important question to ask is which specific subset of these patient would most benefit from additional PMRT. Despite clinical disease stage before NAC, are there any other factors (such as ER/PR status, or pathologic tumor stage after NAC) will influence the patient selection for PMRT among these ypN0 women? A combined analysis of two National Surgical Adjuvant Breast and Bowel Project (NSABP) trials (B18 and B-27) concluded that in addition to initial tumor characteristic before NAC, pathologic response in the breast and the axillary lymph nodes had a major impact on the rates and patterns of LRR; so the pathologic tumor stage and nodal status after NAC might be useful factors for predicting the optimal use of postmastectomy radiation in patients treated with preoperative chemotherapy [23]. Other studies reported that lymphovascular invasion (LVI) was another possible effect modifier that can aid in selecting patients who have a ypN0 following NAC but would benefit from PMRT $[9,24]$. Based on our findings, it appears that patients with more locally advanced disease at presentation (clinical IIIB/IIIC disease or T3/T4 tumor), or with residual invasive breast tumor after NAC (who did not achieve a pCR in the breast) may benefit PMRT as part of their adjuvant therapy even if they downstaged to ypN0 following NAC. However, despite our best attempt to reduce the potential bias and confounding effects from NCDB and the retrospective nature of our study, definitive evidence from randomized controlled trials like NSABP B-51 are required to confirm our results, or identify further subsets of patients who may benefit from PMRT.

Despite several strengths of this study including its multicenter large sample size, refined subgroup analyses, sensitivity analysis using multiple imputation and propensity score matching, several limitations should be acknowledged. First, the NCDB has no recurrence data, so we can not affirm a lack of benefit from PMRT for some subgroups of women simply based on OS alone. This is especially the case for patients with earlier clinical stage disease, where disease control and free from recurrence would more likely be the primary endpoint of interest. Second, the NCDB suffers from lack of HER2 status and detailed histological evaluation including the information of LVI, Ki67, and the incompleteness of ER/PR status in a small portion of patients. All these factors are known to have prognostic value and can predict treatment response. Third, because of the limited number of patients (reduced statistical power) in several subgroup analyses, we cannot conclude lack of survival benefit from PMRT, particularly for patients who were diagnosed before 41 years or after 60 years. Finally, although our study has a median followup time of 56.0 months, longer follow-up might help explore other subsets of women who can benefit from PMRT, especially for low-risk patients.

\section{CONCLUSIONS}

In conclusion, we provided important evidence that among clinically node-positive, stage II-III breast cancer patients with ypN0 following NAC, PMRT can improve overall survival in patients with clinical T3/T4 tumor or stage IIIB/IIIC disease, and in patients with residual invasive breast tumor after NAC. Our study may help oncologists to recommend PMRT for selected patients who downstaged to ypN0 following NAC. Results from further prospective studies such as the ongoing NSABP B-51 trial are needed, in order to confirm our findings and define other specific subgroups of women with pathological negative nodes following NAC who would benefit from PMRT, particularly in the relatively low-risk patients.

\section{ACKNOWLEDGMENTS}

The data used in the study was derived from a deidentified NCDB file. The American College of Surgeons and the Commission on Cancer have not verified and are not responsible for the analytic or statistical methodology used or the conclusions drawn from these data by the investigator. 


\section{GRANT SUPPORT}

This study was supported by grant from the Natural Science Foundation of Guangdong Province (2014A030310507); and the Key Laboratory of Malignant Tumor Molecular Mechanism and Translational Medicine of Guangzhou Bureau of Science and Information Technology ([2013]163); and the Key Laboratory of Malignant Tumor Gene Regulation and Target Therapy of Guangdong Higher Education Institutes (KLB09001).

\section{CONFLICTS OF INTEREST}

The authors have declared no conflicts of interest.

\section{REFERENCES}

1. Kyndi M, Sorensen FB, Knudsen H, Overgaard M, Nielsen HM and Overgaard J. Estrogen receptor, progesterone receptor, HER-2, and response to postmastectomy radiotherapy in high-risk breast cancer: the Danish Breast Cancer Cooperative Group. J Clin Oncol. 2008; 26:14191426.

2. Goldhirsch A, Ingle JN, Gelber RD, Coates AS, Thurlimann B and Senn HJ. Thresholds for therapies: highlights of the St Gallen International Expert Consensus on the primary therapy of early breast cancer 2009. Ann Oncol. 2009; 20:1319-1329.

3. Recht A, Edge SB, Solin LJ, Robinson DS, Estabrook A, Fine RE, Fleming GF, Formenti S, Hudis C, Kirshner JJ, Krause DA, Kuske RR, Langer AS, et al. Postmastectomy radiotherapy: clinical practice guidelines of the American Society of Clinical Oncology. J Clin Oncol. 2001; 19:15391569.

4. Huang EH, Tucker SL, Strom EA, McNeese MD, Kuerer HM, Buzdar AU, Valero V, Perkins GH, Schechter NR, Hunt KK, Sahin AA, Hortobagyi GN and Buchholz TA. Postmastectomy radiation improves local-regional control and survival for selected patients with locally advanced breast cancer treated with neoadjuvant chemotherapy and mastectomy. J Clin Oncol. 2004; 22:4691-4699.

5. Buchholz TA, Tucker SL, Masullo L, Kuerer HM, Erwin J, Salas J, Frye D, Strom EA, McNeese MD, Perkins G, Katz A, Singletary SE, Hunt KK, Buzdar AU and Hortobagyi GN. Predictors of local-regional recurrence after neoadjuvant chemotherapy and mastectomy without radiation. J Clin Oncol. 2002; 20:17-23.

6. Wright JL, Takita C, Reis IM, Zhao W, Saigal K, Wolfson A, Markoe A, Moller M and Hurley J. Predictors of locoregional outcome in patients receiving neoadjuvant therapy and postmastectomy radiation. Cancer. 2013; 119:16-25.

7. Buchholz TA, Lehman CD, Harris JR, Pockaj BA, Khouri N, Hylton NF, Miller MJ, Whelan T, Pierce LJ, Esserman LJ, Newman LA, Smith BL, Bear HD and Mamounas EP.
Statement of the science concerning locoregional treatments after preoperative chemotherapy for breast cancer: a National Cancer Institute conference. J Clin Oncol. 2008; 26:791-797.

8. Le Scodan R, Selz J, Stevens D, Bollet MA, de la Lande B, Daveau C, Lerebours F, Labib A and Bruant S. Radiotherapy for stage II and stage III breast cancer patients with negative lymph nodes after preoperative chemotherapy and mastectomy. Int J Radiat Oncol Biol Phys. 2012; 82:e17.

9. Fowble BL, Einck JP, Kim DN, McCloskey S, Mayadev J, Yashar C, Chen SL and Hwang ES. Role of postmastectomy radiation after neoadjuvant chemotherapy in stage II-III breast cancer. Int J Radiat Oncol Biol Phys. 2012; 83:494503.

10. Shim SJ, Park W, Huh SJ, Choi DH, Shin KH, Lee NK, Suh CO, Keum KC, Kim YB, Ahn SD, Kim SS, Ha SW, Chie EK, Kim K, Shin HS, Kim JH, et al. The role of postmastectomy radiation therapy after neoadjuvant chemotherapy in clinical stage II-III breast cancer patients with pN0: a multicenter, retrospective study (KROG 1205). Int J Radiat Oncol Biol Phys. 2014; 88:65-72.

11. McGuire SE, Gonzalez-Angulo AM, Huang EH, Tucker SL, Kau SW, Yu TK, Strom EA, Oh JL, Woodward WA, Tereffe W, Hunt KK, Kuerer HM, Sahin AA, et al. Postmastectomy radiation improves the outcome of patients with locally advanced breast cancer who achieve a pathologic complete response to neoadjuvant chemotherapy. Int J Radiat Oncol Biol Phys. 2007; 68:1004-1009.

12. Beriwal S, Shinde A, Rajagopalan MS, Kannan N, Heron DE and Deutsch M. Recommendations for post-mastectomy radiation therapy after neo-adjuvant chemotherapy: an International Survey of Radiation Oncologists. Breast J. 2013; 19:683-684.

13. Fritz A PC, Jack A, Shanmugaratnam K, Sobin L, Parkin DM, Whelan S. (2000). International Classification of Diseases for Oncology (ICD-O) 3. Geneva: World Health Organization;.

14. Klebanoff MA and Cole SR. Use of multiple imputation in the epidemiologic literature. Am J Epidemiol. 2008; 168:355-357.

15. Janssen KJ, Donders AR, Harrell FE, Jr., Vergouwe Y, Chen Q, Grobbee DE and Moons KG. Missing covariate data in medical research: to impute is better than to ignore. J Clin Epidemiol. 2010; 63:721-727.

16. Liao SG, Lin Y, Kang DD, Chandra D, Bon J, Kaminski N, Sciurba FC and Tseng GC. Missing value imputation in high-dimensional phenomic data: imputable or not, and how? BMC Bioinformatics. 2014; 15:346.

17. Little RJ, Rubin DB. (2002). Statistical Analysis with Missing Data (Second Edition). (New York: John Wiley \& Sons;).

18. Normand ST, Landrum MB, Guadagnoli E, Ayanian JZ, Ryan TJ, Cleary PD and McNeil BJ. Validating 
recommendations for coronary angiography following acute myocardial infarction in the elderly: a matched analysis using propensity scores. J Clin Epidemiol. 2001; 54:387398.

19. R Core Team 2014. A language and environment for statistical computing. $\mathrm{R}$ Foundation for Statistical Computing, Vienna, Austria. URL http://www.R-project. org/.

20. Gralow JR, Burstein HJ, Wood W, Hortobagyi GN, Gianni L, von Minckwitz G, Buzdar AU, Smith IE, Symmans WF, Singh B and Winer EP. Preoperative therapy in invasive breast cancer: pathologic assessment and systemic therapy issues in operable disease. J Clin Oncol. 2008; 26:814-819.

21. Mamounas TP. Predicting locoregional recurrence after neoadjuvant chemotherapy in patients with breast cancer. Clin Adv Hematol Oncol. 2013; 11:175-177.

22. Hardenbergh PH, Bentel GC, Prosnitz LR and Marks LB. Postmastectomy radiotherapy: toxicities and techniques to reduce them. Semin Radiat Oncol. 1999; 9:259-268.

23. Mamounas EP, Anderson SJ, Dignam JJ, Bear HD, Julian TB, Geyer CE, Jr., Taghian A, Wickerham DL and Wolmark N. Predictors of locoregional recurrence after neoadjuvant chemotherapy: results from combined analysis of National Surgical Adjuvant Breast and Bowel Project B-18 and B-27. J Clin Oncol. 2012; 30:3960-3966.

24. Huang EH, Tucker SL, Strom EA, McNeese MD, Kuerer HM, Hortobagyi GN, Buzdar AU, Valero V, Perkins GH, Schechter NR, Hunt KK, Sahin AA and Buchholz TA. Predictors of locoregional recurrence in patients with locally advanced breast cancer treated with neoadjuvant chemotherapy, mastectomy, and radiotherapy. Int J Radiat Oncol Biol Phys. 2005; 62:351-357. 